\title{
High levels of glioma tumor suppressor candidate region gene 1 predicts a poor prognosis for prostate cancer
}

\author{
XIAOMING MA ${ }^{1,2^{*}}$, TAO DU ${ }^{2,3^{*}}$, DINGJUN ZHU ${ }^{1,2^{*}}$, XIANJU CHEN $^{1}$, YIMING LAI ${ }^{1}$, WANHUA WU ${ }^{1}$,

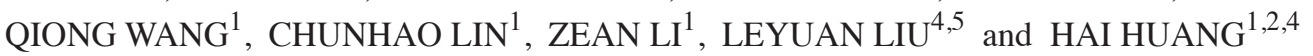 \\ ${ }^{1}$ Department of Urology; ${ }^{2}$ Guangdong Provincial Key Laboratory of Malignant Tumor Epigenetics and Gene Regulation; \\ ${ }^{3}$ Department of Obstetrics and Gynecology, Sun Yat-Sen Memorial Hospital, Sun Yat-Sen University, \\ Guangzhou, Guangdong 510120, P.R. China; ${ }^{4}$ Center for Translational Cancer Research, \\ Texas A\&M Institute of Biosciences and Technology; ${ }^{5}$ Department of Molecular and Cellular Medicine, \\ College of Medicine, Texas A\&M University, Houston, TX 77030, USA
}

Received October 24, 2017; Accepted June 8, 2018

DOI: $10.3892 / \mathrm{ol} .2018 .9490$

\begin{abstract}
Glioma tumor suppressor candidate region gene 1 (GLTSCR1) is associated with the progression of oligodendroglioma. However, there has been little study of GLTSCR 1 in prostate cancer. In the present study, the association between the expression of GLTSCR1, and the progression and prognosis of tumors in patients with prostate cancer was assessed. An immunohistochemical analysis was performed using a human tissue microarray for GLTSCR1 at the protein expression level and the immunostaining results were evaluated against clinical variables of patients with prostate cancer. Subsequently, The Cancer Genome Atlas (TCGA) was used to validate the analysis results at the mRNA level and to study the prognostic value of GLTSCR1 in prostate cancer. Immunohistochemistry and TCGA data analysis revealed that GLTSCR1 expression in the prostate cancer tissues was significantly higher than that in the benign prostate tissues (immunoreactivity score, $\mathrm{P}=0.015$; mRNA levels: cancer, $447.7 \pm 6.45$ vs. benign, $343.5 \pm 4.21$; $\mathrm{P}<0.001$ ). Additionally, the increased GLTSCR1 protein expression was associated with certain clinical variables in the prostate cancer tissues, including advanced clinical stage
\end{abstract}

Correspondence to: Professor Hai Huang, Department of Urology, Sun Yat-Sen Memorial Hospital, Sun Yat-Sen University, 107 West Yanjiang Road, Guangzhou, Guangdong 510120, P.R. China E-mail: huanghai257@126.com

Professor Leyuan Liu, Center for Translational Cancer Research, Texas A\&M Institute of Biosciences and Technology, Texas A\&M University, 2121 West Holcombe Boulevard, Houston, TX 77030, USA

E-mail: 1liu@ibt.tamhsc.edu

${ }^{*}$ Contributed equally

Key words: prostate cancer, glioma tumor suppressor candidate region gene 1, clinicopathological characteristic, biochemical recurrence-free survival, prognosis
$(\mathrm{P}<0.001)$, enhanced tumor invasion $(\mathrm{P}=0.003)$, lymph node metastasis $(\mathrm{P}=0.003)$ and distant metastasis $(\mathrm{P}=0.001)$. TCGA data revealed similar results, demonstrating that the upregulation of GLTSCR1 mRNA expression was associated with the Gleason score $(\mathrm{P}<0.001)$, enhanced tumor invasion $(\mathrm{P}=0.011)$, lymph node metastasis $(\mathrm{P}=0.001)$ and distant metastasis $(\mathrm{P}=0.002)$. Furthermore, Kaplan-Meier analysis suggested that among all patients, high GLTSCR1 expression indicated a decreased overall survival $(\mathrm{P}=0.028)$ and biochemical recurrence $(\mathrm{BCR})$-free survival $(\mathrm{P}=0.004)$, compared with patients with low GLTSCR1 expression. Finally, multivariate analysis revealed that the expression of GLTSCR1 was an independent predictor of poor BCR-free survival $(\mathrm{P}=0.049)$. The present study suggested that the increased expression of GLTSCR1 was associated with the progression of prostate cancer. Furthermore, GLTSCR1 may be a novel biomarker that is able to predict the clinical outcome in prostate cancer patients.

\section{Introduction}

Prostate cancer is the second leading cause of cancer-associated mortality among males in the United States (1). It is estimated that in 2018 there will be $\sim 161,360$ newly diagnosed cases of prostate cancer in the United States and 26,730 of them will result in mortality (2). In China, the morbidity and mortality rates of prostate cancer are also continuously increasing (3). Prostate cancer is a disease presenting with a number of heterogeneous symptoms. Numerous factors are recognized as risk factors of prostate cancer, including race, age and genetic mutations. These risk factors are associated with the tumorigenesis, progression and prognosis of prostate cancer (4). The tumorigenesis and progression of this disease are difficult to assess, and it is particularly difficult to provide an accurate prognosis. Radical prostatectomy and radiation therapy are basic strategies used to treat localized prostate cancer (5); however, following radical prostatectomy or radiation therapy, $20 \%$ of patients experience biochemical recurrence (BCR) and require additional treatment (6). Due to the heterogeneous nature of prostate cancer, every patient requires personalized treatment regimens. Therefore, it is important to identify the 
state of progression and predict the prognosis of prostate cancer in each individual. To predict the disease outcome, serum levels of prostate-specific antigen (PSA), tumor invasion, Gleason Score, and lymph node and distant metastasis have been used in a number of combinations $(7,8)$. However, this methodology is not sufficient to confirm diagnosis and it remains difficult to determine the disease outcome using the limited clinicopathological data available. For example, PSA is universally used in screening, detecting and predicting the prognosis of prostate cancer (9); however, numerous false positives have been reported as a result of PSA screening (10). Therefore, a novel and accurate biomarker for the diagnosis and prognosis of prostate cancer is required.

Microtubule-associated protein 1 small form (MAP1S) is a marker of autophagy, and it serves a notable function in the course of autophagosomal biogenesis and degradation (11). A previous study hypothesized that patients with prostate cancer with a low expression level of MAP1S experience a poor prognosis (12). Using The Cancer Genome Atlas (ATLAS) database, it was identified that glioma tumor suppressor candidate region gene 1 (GLTSCR1) was moderately associated with MAP1S at the mRNA expression level (Fig. 1).

GLTSCR1 is associated with the development and progression of oligodendroglioma $(13,14)$. It is located on 19q13.33 and exhibits moderate expression levels in the brain, heart, skeletal muscle, placenta and pancreas, and low expression levels in the liver, lungs and kidney $(13,15)$. The current literature has only suggested that GLTSCR1 is involved in oligodendroglioma $(13,14)$. To the best of our knowledge, there are no studies describing the role of GLTSCR1 in prostate cancer. Previous studies have speculated that there are a number of susceptibility loci for prostate cancer aggressiveness on chromosome 19q13 $(15,16)$. Notably, as aforementioned, GLTSCR1 is located on 19q13. Therefore, according to the association between GLTSCR1 and MAP1S identified via TCGA, the location of GLTSCR1 on chromosome 19q13 and the function of GLTSCR1 in oligodendroglioma, it was hypothesized that GLTSCR1 may be associated with prostate cancer.

The present study investigated the expression level of GLTSCR1 in prostate cancer, and determined the association between GLTSCR1 expression and the clinicopathological features and prognosis of patients with prostate cancer.

\section{Materials and methods}

Patients and tissue samples. In order to perform an immunohistochemical analysis, a tissue microarray (TMA; $n=80$ ), including 4 prostate tissues, 3 adjacent normal prostate tissues and 73 prostate cancer tissues was purchased (cat. no. PR803c; Alenabio Biotechnology Ltd., Xi'an, China), along with the detailed clinicopathological information of each sample including the Gleason score, TNM staging and the clinical stage. The staging met the standards required by previous studies $(17,18)$. Samples from patients who had received radiotherapy or chemotherapy prior to their surgery were excluded from the present study. To study the expression of GLTSCR1 at the mRNA level and complete a survival analysis, clinicopathological features from TCGA database, a freely opened public platform, is a source for abundant cancer-related data (19), including 499 prostate cancer tissues and 52 normal prostate tissues, were also gathered. These features are summarized in Table I.

Immunohistochemistry. The samples were fixed in $10 \%$ neutral buffered formalin overnight at room temperature, and embedded in paraffin. The paraffin-embedded tissues were sliced into $4-\mu \mathrm{m}$ thick sections, dewaxed in xylene and rehydrated in a descending alcohol series (100, 100, 95, $95,90,80$ and $70 \%$, for $2 \mathrm{~min}$ each) at room temperature. Sections were subjected to peroxidase immunohistochemistry staining employing a DAKO EnVision system (Dako; Agilent Technologies, Inc., Santa Clara, CA, USA). The sections were subjected to antigen retrieval by peroxidase with $0.01 \mathrm{M}$ citrates (cat. no. AR0024; Boster Biological Technology, Wuhan, China) in a microwave oven on high for $\sim 6 \mathrm{~min}$, moderate for $\sim 6 \mathrm{~min}$ and blocked with goat serum for $30 \mathrm{~min}$. The immunohistochemical staining of tissue microarray was conducted using the UltraSensitive ${ }^{\mathrm{TM}} \mathrm{SP}$ (Mouse/Rabbit) IHC kit (cat no. KIT-0305; MX Biotechnologies, Fuzhou, China). Next, the sections were incubated with the corresponding rabbit polyclonal antibody against GLTSCR1 (1:600; cat. no. bs-14278R; Bioss Antibodie, Inc., Woburn, MA, USA), overnight at $4^{\circ} \mathrm{C}$. Subsequently, the sections were incubated with the HRP-labeled secondary antibody [dilution, 1:200; UltraSensitive SP (Mouse/Rabbit) IHC kit; cat no. KIT-0305; MX Biotechnologies] marked by avidin for $30 \mathrm{~min}$ at room temperature. Then peroxidase-labeled polymer (incubated with the $50 \mu \mathrm{l}$ for $15 \mathrm{~min}$ at room temperature) and substrate-chromogen (incubated with the $100 \mu \mathrm{l}$ for $2 \mathrm{~min}$ at room temperature) were used so as to observe the staining of the target protein. Sections undergoing the same procedure omitting the corresponding antibody served as the negative controls.

Assessment of immunostaining results. The stained slides were scanned by Aperio ImageScope (Aperio CS; Leica Microsystems, Inc., Buffalo Grove, IL, USA). Then these slides were scored by two independent experienced pathologists in a blinded manner, (Department of Pathology Diagnosis, Sun Yat-Sen Memorial Hospital, Guangdong, China), and any disagreements were resolved by a re-examination of the section by the pathologists in order to obtain an agreed conclusion. The immunolabeling of tumor cells was assessed. The number of positively stained cells in five representative microscopic fields at a magnification of $x 400$ was counted and the percentage of positive cells was calculated. Cytoplasmic staining was considered indicative of a positive signal on the basis of the antibody specification sheet. The staining intensity and percentage were used for semi-quantitative scoring of the expression intensity in each case, as described by a previous study (20). The staining intensity was scored as follows: Negative, weak, moderate and strong staining were scored as $0,1,2$ and 3 points, respectively. The percentage scoring of immunoreactive tumor cells was classified according to the following criteria: $>75 \%$, 4 points; $51-75 \%, 3$ points; $26-50 \%$, 2 points; $6-25 \%, 1$ point; $<5 \%, 0$ points. Multiplying immunostaining intensity and immunostaining percentage scores of each sample provided the final immunoreactivity score (IRS) for each sample. In the immunohistochemistry score, 0-1 was considered as negative, 2-4 as low expression (+), 5-8 as 
Table I. Clinical characteristics of all patients.

Experiment type

\begin{tabular}{lcr}
\cline { 2 - 3 } Clinical characteristic & TMA & TCGA \\
\hline Tissue type & & \\
Prostate cancer & 73 & 499 \\
Normal & 7 & 52 \\
Serum PSA levels, ng/ml & & \\
$<4$ & na & 413 \\
$\geq 4$ & na & 27 \\
Gleason score & & \\
$<7$ & na & 44 \\
$=7$ & na & 247 \\
$>7$ & na & 206 \\
Lymph node metastasis & & \\
N0 & 60 & 344 \\
N1 & 12 & 80 \\
Distant metastasis & & \\
M0 & 58 & 455 \\
M1 & 14 & 3 \\
\hline
\end{tabular}

TCGA, the cancer genome atlas; TMA, tissue microarray; PSA, prostate-specific antigen; na, not applicable; $\mathrm{N}$, node; $\mathrm{M}$, metastasis.

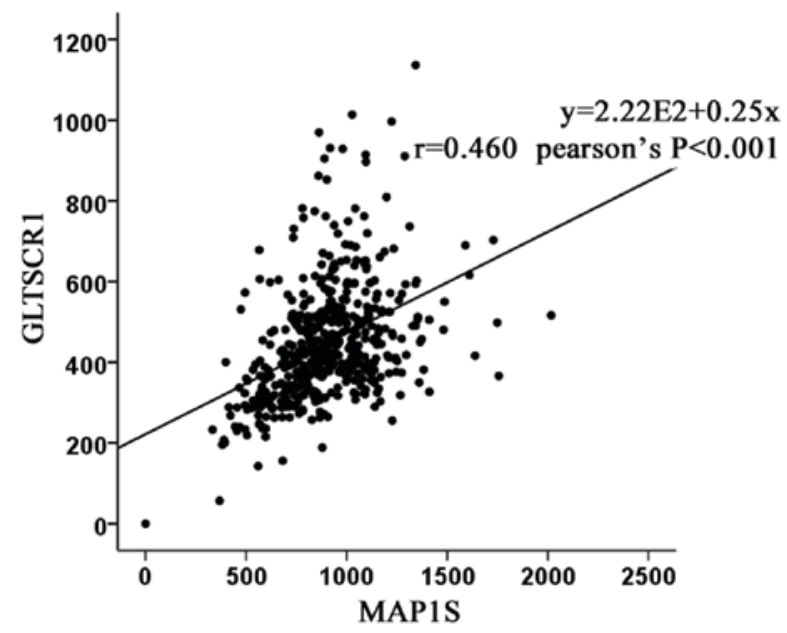

Figure 1. Analysis of the association between GLTSCR1 and MAP1S based on The Cancer Genome Atlas. GLTSCR1 was moderately associated with MAP1S at the mRNA expression level. $r=0.460, \mathrm{P}<0.001$. GLTSCR1, glioma tumor suppressor candidate region gene 1; MAP1S, microtubule-associated protein 1 small form.

moderate expression (++), and 9-12 as high expression (+++). Therefore, samples with negative expression and low expression ( $\leq 4$ points) were designated as the low expression group, whilst the moderate expression and high expression samples ( $>4$ points) were designated as the high expression group.

Statistical analysis. SPSS software (version 22.0; IBM Corp., Armonk, NY, USA) was used to statistically analyze all results. An analysis of variance (ANOVA) and least significance difference (LSD) post hoc test were used for analysis of the association between Gleason score and GLTSCR1 expression. Fisher's exact test, Student's t-test and Pearson's $\chi^{2}$ test were used for analysis of the association between other clinicopathological features and GLTSCR1 expression. The Kaplan-Meier method was used to analyze the overall survival rate, and a log-rank test was used to evaluate the differences in survival rate. Cox's proportional hazard regression model was performed for univariate and multivariate survival analysis. Adjusted hazard ratios (HRs) and corresponding 95\% confidence intervals (CIs) reflect the relative risks of mortality. $\mathrm{P}<0.05$ was considered to indicate a statistically significant difference.

\section{Results}

GLTSCR1 protein is upregulated in human prostate cancer and is associated with certain clinicopathological parameters in patients with prostate cancer. GLTSCR1 protein expression was detected using a TMA $(\mathrm{n}=80)$ via immunohistochemistry (Table II), including 3 adjacent normal prostate tissues, 4 normal prostate tissues and 73 prostate cancer tissues. As presented in Fig. 2A, GLTSCR1 immunostaining occurred moderately and weakly in both the cytoplasm of prostate cancer cells and normal prostate tissues, but the expression level of GLTSCR1 in prostate cancer tissues was significantly increased compared with that in normal prostate tissue (IRS, prostate cancer, $4.82 \pm 0.99$ versus normal, $3.86 \pm 0.69 ; \mathrm{P}=0.015$; Fig. 2B). Of the 73 prostate cancer samples, 26 (35.6\%) exhibited low GLTSCR1 expression, while 47 (64.4\%) exhibited high GLTSCR1 expression.

Subsequently, immunostaining results were statistically analyzed using the clinicopathological information obtained from the TMA. The results revealed that the high expression of GLTSCR1 protein was significantly associated with advanced clinical stage $(\mathrm{P}<0.001)$, enhanced tumor invasion $(\mathrm{P}=0.003)$, lymph node metastasis $(\mathrm{P}=0.003)$ and distant metastasis $(\mathrm{P}=0.001)$. However, no association was detected between GLTSCR1 expression level and age ( $\mathrm{P}>0.05$; Table II).

High expression of GLTSCRI is associated with the aggressive progression and poor prognosis of prostate cancer. To validate the results of the immunohistochemistry analysis, data from TCGA were used to assess the mRNA expression of GLTSCR1 in 52 normal prostate tissues and 499 prostate cancer tissues. As presented in Table II, the cancer patients were divided into 3 groups (GS<7, GS=7 and GS $>7$ ) and results were compared using ANOVA and LSD tests $(\mathrm{P}<0.001$ for ANOVA analysis of the 3 groups); the results revealed that GLTSCR1 was upregulated in PCa tissues and was positively associated with the Gleason score, and enhanced tumor invasion $(\mathrm{P}=0.011)$, lymph node metastasis $(\mathrm{P}=0.001)$ and distant metastasis $(\mathrm{P}=0.002)$. However, high GLTSCR1 expression levels were not associated with age $(\mathrm{P}>0.05$; Table II).

Furthermore, the prognostic value of GLTSCR1 relative to the survival time of patients with prostate cancer was evaluated using Kaplan-Meier survival plots. As presented in Fig. 3, the overall survival and BCR-free survival times of all prostate cancer patients with high GLTSCR1 expression were significantly shorter than those with low GLTSCR1 
Table II. Association between GLTSCR1 expression and clinicopathological characteristics in patients with prostate cancer.

\begin{tabular}{|c|c|c|c|c|c|c|c|}
\hline \multirow[b]{2}{*}{ Clinical features } & \multicolumn{4}{|c|}{ TMA (Pearson's $\chi^{2}$ tests) } & \multicolumn{3}{|c|}{ TCGA (Student's t-tests) } \\
\hline & Cases & Low, n (\%) & High, n (\%) & P-value & Cases & Mean \pm SD & P-value \\
\hline \multicolumn{8}{|l|}{ Tissue type } \\
\hline Prostate cancer & 73 & $26(35.6)$ & $47(64.4)$ & \multirow[t]{2}{*}{$0.015^{\mathrm{a}}$} & 499 & $447.7 \pm 6.45$ & \multirow[t]{2}{*}{$<0.001^{t}$} \\
\hline Benign & 7 & $6(85.7)$ & $1(14.3)$ & & 52 & $343.5 \pm 14.21$ & \\
\hline \multicolumn{8}{|l|}{ Age } \\
\hline$<66$ & 24 & $11(45.8)$ & $13(54.2)$ & \multirow[t]{2}{*}{0.202} & 354 & $453.79 \pm 146.85$ & \multirow[t]{2}{*}{0.111} \\
\hline$\geq 66$ & 49 & $15(30.6)$ & $34(69.4)$ & & 143 & $431.08 \pm 135.44$ & \\
\hline \multicolumn{8}{|l|}{ PSA level (ng/ml) } \\
\hline$\leq 4$ & na & na & na & \multirow[t]{2}{*}{ na } & 413 & $446.73 \pm 143.75$ & \multirow[t]{2}{*}{0.723} \\
\hline$>4$ & na & na & na & & 27 & $456.83 \pm 135.92$ & \\
\hline \multicolumn{8}{|l|}{ Gleason score } \\
\hline$<7$ & na & na & na & \multirow[t]{3}{*}{ na } & 44 & $427.34 \pm 114.67$ & \multirow[t]{3}{*}{$<0.001^{t}$} \\
\hline$=7$ & na & na & na & & 247 & $419.46 \pm 128.84$ & \\
\hline$>7$ & na & na & na & & 206 & $484.84 \pm 158.06$ & \\
\hline \multicolumn{8}{|l|}{ Pathological grade } \\
\hline$\leq 2$ & 23 & $6(26.1)$ & $17(73.9)$ & \multirow[t]{2}{*}{0.304} & na & na & \multirow[t]{2}{*}{ na } \\
\hline$>2$ & 44 & $17(38.6)$ & $27(61.4)$ & & na & na & \\
\hline \multicolumn{8}{|l|}{ Clinical stage } \\
\hline I-II & 45 & $24(53.3)$ & $21(46.7)$ & \multirow[t]{2}{*}{$<0.001^{\mathrm{b}}$} & na & na & \multirow[t]{2}{*}{ na } \\
\hline III-IV & 27 & $2(7.4)$ & $25(92.6)$ & & na & na & \\
\hline \multicolumn{8}{|l|}{ Tumor invasion } \\
\hline $\mathrm{T} 1-\mathrm{T} 2$ & 48 & $23(47.9)$ & $25(52.1)$ & \multirow[t]{2}{*}{$0.003^{\mathrm{b}}$} & $177(\mathrm{~T} 1)$ & $437.32 \pm 131.93$ & \multirow[t]{2}{*}{$0.011^{\mathrm{a}}$} \\
\hline T3-T4 & 24 & $3(12.5)$ & $21(87.5)$ & & 229 (T2-T4) & $474.09 \pm 152.16$ & \\
\hline \multicolumn{8}{|c|}{ Lymph node metastasis } \\
\hline No & 60 & $26(43.3)$ & $34(56.7)$ & \multirow[t]{2}{*}{$0.003^{\mathrm{b}}$} & 344 & $440.49 \pm 139.44$ & \multirow[t]{2}{*}{$0.001^{\mathrm{b}}$} \\
\hline N1 & 12 & $0(0.0)$ & $12(100.0)$ & & 80 & $503.48 \pm 174.50$ & \\
\hline \multicolumn{8}{|l|}{ Distant metastasis } \\
\hline M0 & 58 & $26(44.8)$ & $32(55.2)$ & \multirow[t]{2}{*}{$0.001^{\mathrm{b}}$} & 455 & $448.68 \pm 141.05$ & \multirow[t]{2}{*}{$0.002^{\mathrm{b}}$} \\
\hline M1 & 14 & $0(0.0)$ & $14(100.0)$ & & 3 & $710.18 \pm 381.93$ & \\
\hline
\end{tabular}

${ }^{\mathrm{a}} \mathrm{P}<0.05,{ }^{\mathrm{b}} \mathrm{P}<0.01$. GLTSCR 1, Glioma Tumor Suppressor Candidate Region Gene 1; PSA, prostate-specific antigen; TCGA, the Cancer Genome Atlas; na, not applicable; T, tumor; N, node; M, metastasis; SD, standard deviation.
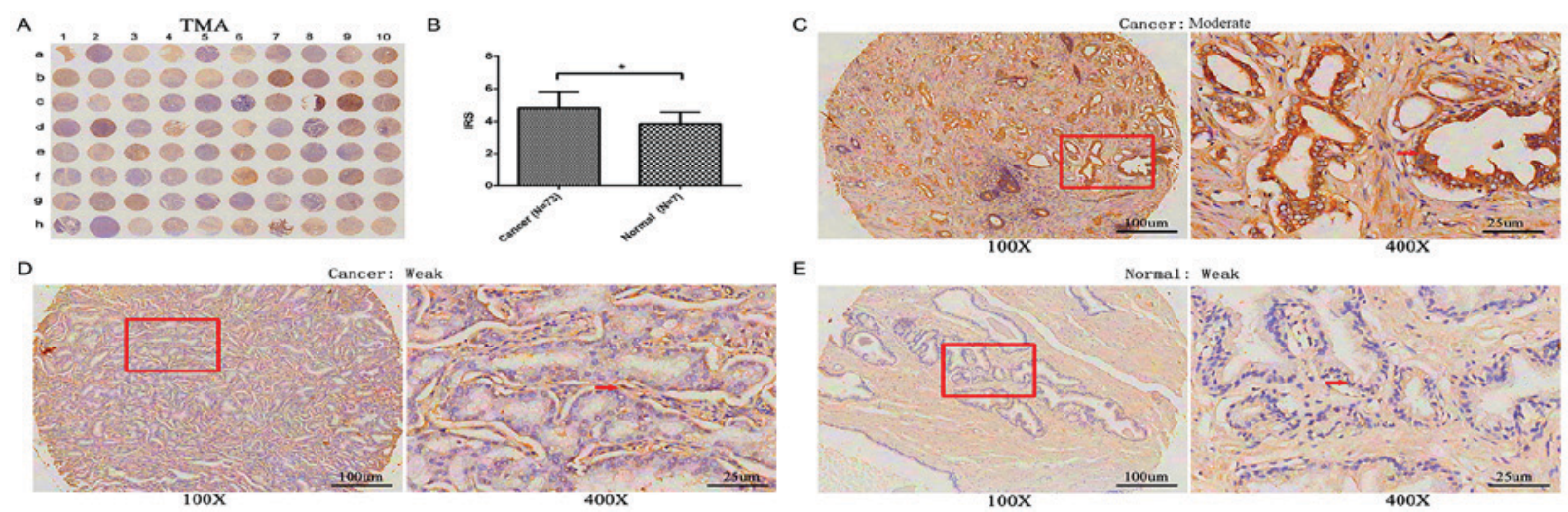

Figure 2. Immunohistochemical staining for GLTSCR1 in prostate cancer and normal prostate TMA samples. (A) A full view of the immunohistochemistry staining for GLTSCR1 in the TMA cohort. (B) The IRS of GLTSCR1 in prostate cancer were higher than those in normal prostate tissues (prostate cancer $=4.82 \pm 0.99$ vs. normal $=3.86 \pm 0.69 ; \mathrm{P}=0.015$ ). The immunohistochemistry staining indicated that GLTSCR1 immunostaining mainly occurred in the cytoplasm of prostate cancer, the intensity of GLTSCR1 immunostaining was (C) moderate and (D) weak in prostate cancer tissues, and (E) weak in normal prostate tissues. TMA, tissue microarray; GLTSCR1, glioma tumor suppressor candidate region gene 1; IRS, immunoreactivity score. "P<0.05. 
A

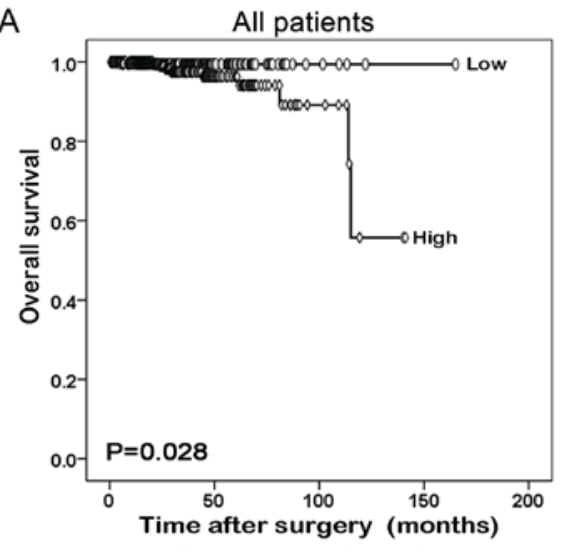

C

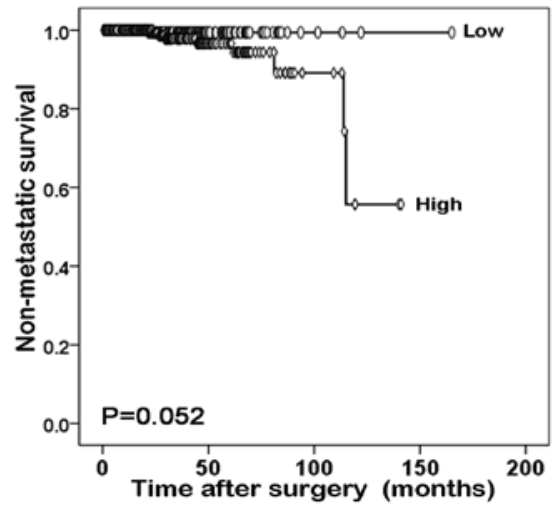

B

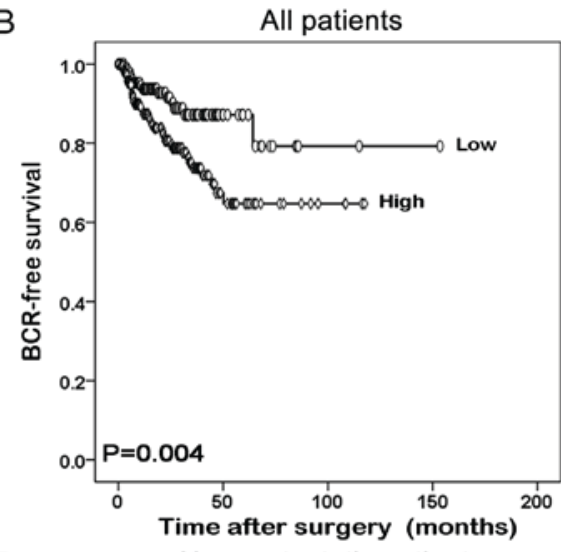

D

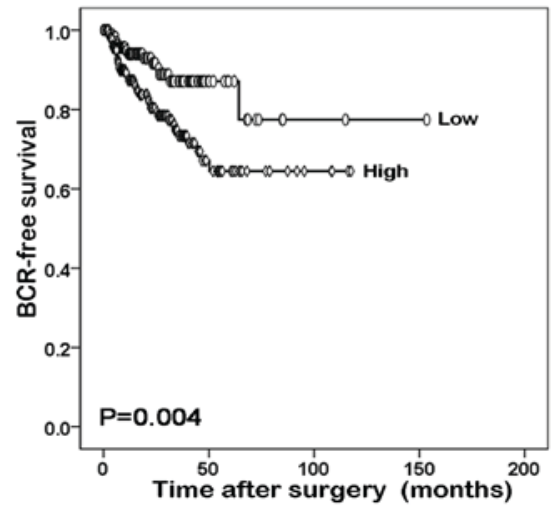

Figure 3. Low expression of GLTSCR1 predicts improved prognosis in prostate cancer patients. The Kaplan-Meier method was performed to evaluate the difference in (A) overall and (B) BCR-free survival between high and low expression levels of GLTSCR1 in all patients. The Kaplan-Meier method was performed to evaluate the difference in (C) non-metastatic survival and (D) BCR-free survival between high and low expression levels of GLTSCR1 in non-metastatic patients. BCR, biochemical recurrence; GLTSCR1, glioma tumor suppressor candidate region gene 1.

expression $(\mathrm{P}=0.028$ and $\mathrm{P}=0.004$, respectively). The same result was obtained in the BCR-free survival of patients with non-metastatic prostate cancer $(\mathrm{P}=0.004)$. However, the different GLTSCR1 expression was not significant in the non-metastatic survival of patients with non-metastatic prostate cancer.

GLTSCR1 serves as an independent prognostic factor for the survival of patients with prostate cancer. Finally, using Cox's proportional hazards model on data obtained from TCGA, the possibility that GLTSCR 1 is an independent prognostic factor for the survival of prostate cancer was evaluated. The univariate analysis revealed that GLTSCR 1 expression was a significant prognostic factor for BCR-free survival in patients with prostate cancer (HR, 2.279; 95\% CI, 1.284-4.047; $\mathrm{P}=0.005)$, alongside the Gleason score, PSA level, tumor invasion and lymph node metastasis (all $\mathrm{P}<0.05$; Table III). Multivariate analysis revealed that high GLTSCR1 expression was a significant independent prognostic factor in prostate cancer (HR, 1.829; 95\% CI, 1.002-3.339; $\mathrm{P}=0.049)$, alongside the Gleason score and PSA level (all $\mathrm{P}<0.05$; Table III).

\section{Discussion}

Prostate cancer is a common disease with a high morbidity rate in the USA (21). The disease is characterized by numerous heterogeneous lesions, and the use of presently known biomarkers to determine the state of disease progression and predict the prognosis remains a challenge. Therefore, further studies are required to identify a novel and accurate biomarker to assess the disease stage and predict the outcome for patients. In the present study, it was revealed that the expression of GLTSCR1 in prostate cancer tissue was increased compared with that in normal tissues. Additionally, the GLTSCR1 expression was markedly associated with the progression of prostate cancer. Notably, GLTSCR1 may function as a significant independent prognostic factor in prostate cancer, since high expression of GLTSCR1 was associated with poorer BCR-free survival in patients with prostate cancer.

GLTSCR1 is involved in the development and progression of oligodendroglioma $(13,14)$. Previous investigations demonstrated that on chromosome 19q13, a number of susceptibility loci are associated with the aggressiveness of prostate cancer $(15,16)$. Notably, GLTSCR1 is located on 19q13.33 $(14,15)$, suggesting that GLTSCR1 may be associated with prostate cancer. Using TCGA, the present study identified that GLTSCR1 was moderately associated with MAP1S at the mRNA expression level. MAP1S is a biomarker of autophagy, and a previous study demonstrated that patients with prostate cancer exhibiting low MAP1S expression levels exhibit a poor prognosis $(11,12)$. In light of the aforementioned studies, it was hypothesized that GLTSCR1 may promote prostate cancer progression. Therefore, the association between the expression of GLTSCR1 in prostate cancer tissues, and the clinicopathological parameters and prognosis of patients with prostate cancer was evaluated. 
Table III. Prognostic value of GLTSCR1 expression for the BCR-free survival proportional hazards model.

BCR-free survival

Variable

$\operatorname{HR}(95 \% \mathrm{CI})$

P-value

Univariate analysis

Age, $\geq 60$ vs. $<60$

$1.319(0.773-2.250)$

0.310

PSA level, $>4$ vs. $\leq 4$

$10.426(5.309-20.474)$

$<0.001^{\text {b }}$

Gleason score, $<7$ vs. $=7$ vs. $>7$

$3.175(1.881-5.360)$

$<0.001^{\mathrm{b}}$

Tumor invasion, T1 vs. T2-T4

$3.416(1.714-6.810)$

$<0.001^{\text {b }}$

Distant metastasis, M0 vs. M1

$3.536(0.488-25.641)$

0.212

Lymph node metastasis, N0 vs. N1

$1.879(1.049-3.365)$

0.034

GLTSCR1 expression, low vs. high

$2.279(1.284-4.047)$

$0.005^{\mathrm{b}}$

Multivariate analysis

Age $\geq 60$ vs. $<60$

$1.026(0.594-1.771)$

0.927

PSA level, $>4$ vs. $\leq 4$

$6.093(3.015-12.316)$

$<0.001^{\text {b }}$

Gleason score, $<7$ vs. $=7$ vs. $>7$

$2.326(1.343-4.028)$

$0.003^{\mathrm{b}}$

GLTSCR1 expression, low vs. high

$1.829(1.002-3.339)$

$0.049^{\mathrm{a}}$

${ }^{\mathrm{a}} \mathrm{P}<0.05,{ }^{\mathrm{b}} \mathrm{P}<0.01$. GLTSCR1, Glioma Tumor Suppressor Candidate Region Gene 1; BCR, biochemical recurrence; HR, hazard ratio; CI, confidence interval; PSA, prostate-specific antigen.

In present study, it was revealed that GLTSCR1 protein expression was markedly associated with advanced clinical stage, enhanced tumor invasion, and lymph node and distant metastasis in the prostate cancer tissues. Additionally, it was also identified that the GLTSCR1 RNA expression level in prostate cancer was associated with the Gleason score, enhanced tumor invasion, and lymph node and distant metastasis, based on data from TCGA. Those clinicopathological parameters directly indicated the stage of prostate cancer. Furthermore, it was identified that the patients with prostate cancer exhibiting high expression of GLTSCR1 experienced a significantly shorter overall survival time compared with those with low expression of GLTSCR1 ( $\mathrm{P}=0.028)$. Notably, the results from multivariate analysis revealed that GLTSCR1 was a novel independent prognostic factor in prostate cancer.

According to the present study, GLTSCR1 served a marked function in promoting prostate cancer progression. However, the underlying molecular mechanism by which GLTSCR1 promotes prostate cancer remains unknown. A previous study demonstrated that GLTSCR1 is able to upregulate Pim-2 oncogene (PIM2) in C33A cells (22). PIM2 is a potential target of cancer therapy since blocking the activity of PIM2 may prevent the development of pancreatic cancer and multiple myeloma (23-25). Additionally, the overexpression of PIM2 may promote the tumorigenesis of prostate cancer, and high levels of PIM2 in prostate cancer are associated with an increased risk of biochemical recurrence (26). Furthermore, PIM2 may regulate autophagy, and PIM-2 suppression downregulates autophagy by preventing dissociation of B cell lymphoma-2 from Beclin 1 (27). According to the aforementioned studies, it was hypothesized that GLTSCR1 promotes prostate cancer by upregulating PIM2. However, this hypothesis requires further investigation. As stated earlier, TCGA revealed that GLTSCR1 was correlated with MAP1S at the mRNA expression level in prostate cancer. The present study suggested that low expression of GLTSCR1 is indicative of a good prognosis in patients with prostate cancer. However, a previous study suggested that low expression of MAP1S is indicative of a poor prognosis in patients with prostate cancer (12). Therefore, the results of the two studies are contradictory. This may arise from the character of autophagy, since PIM2 and MAP1S are associated with autophagy $(11,22)$. Autophagy may serve contrary functions in different stages of cancer (28). Therefore, the conclusions drawn require further investigation.

In conclusion, the present study suggested a sensitive and accurate biomarker that may aid in the diagnosis and prognosis of prostate cancer. However, continued study is required to clarify the underlying molecular mechanisms by which GLTSCR1 promotes prostate cancer.

\section{Acknowledgements}

The authors would like to thank Professor Jian Huang for their cooperation in the preparation of this manuscript.

\section{Funding}

The present study was supported by the National Natural Science Foundation of China (grant nos. 81472382 and 81672550), the Guangdong Province Natural Science Foundation (grant no. 2014A030313079), the Guangdong Province Science and Technology for Social Development Project (grant nos. 2017A020215018), the Guangzhou City in 2015 Scientific Research Project (grant no. 201510010298), the International Science and Technology Cooperation Project of Guangdong Province Science and Technology Plan (grant no. 2016A050502020) and the Guangzhou International Science and Technology Cooperation Program (grant no. 201807010087). 


\section{Availability of data and materials}

All data generated or analyzed during this study are included in this published article.

\section{Authors' contributions}

Guarantor of integrity of entire study: XM, TD, DZ, XC, Y, WW, QW, CL, ZL, HH and LL. Study concepts: XM, TD and DZ. Study design: XM, TD, DZ, HH and LL. Literature research: XM, TD and DZ. Experimental studies: XC, YL, WW, QW, CL and ZL. Data acquisition: XC, YL, WW, QW, CL and ZL. Data analysis/interpretation: XC, YL, WW, QW, CLZL, HH and LL. Statistical analysis: XC, YL, WW, QW, CL and ZL. Manuscript preparation: XM, TD and DZ. Manuscript definition of intellectual content: XM, TD, DZ and HH. Manuscript editing: XM, TD and DZ. Manuscript revision/review: HH and LL. Manuscript final version approval: $\mathrm{HH}$ and LL.

\section{Ethics approval and consent to participate}

Ethical approval was obtained for this study from Ethics Committee of Sun Yat-Sen Memorial Hospital, Sun Yat-Sen University.

\section{Patient consent for publication}

Not applicable.

\section{Competing interests}

The authors declare that they have no competing interests.

\section{References}

1. Siegel R, Naishadham D and Jemal A: Cancer statistics, 2013 CA Cancer J Clin 63: 11-30, 2013.

2. Siegel RL, Miller KD and Jemal A: Cancer statistics, 2017. CA Cancer J Clin 67: 7-30, 2017.

3. Center MM, Jemal A, Lortet-Tieulent J, Ward E, Ferlay J, Brawley $\mathrm{O}$ and Bray $\mathrm{F}$ : International variation in prostate cancer incidence and mortality rates. Eur Urol 61: 1079-1092, 2012.

4. Cuzick J, Thorat MA, Andriole G, Brawley OW, Brown PH, Culig Z, Eeles RA, Ford LG, Hamdy FC, Holmberg L, et al: Prevention and early detection of prostate cancer. Lancet Oncol 15: e484-e492, 2014.

5. Abdollah F, Sun M, Schmitges J, Thuret R, Bianchi M, Shariat SF, Briganti A, Jeldres C, Perrotte P, Montorsi F and Karakiewicz PI Survival benefit of radical prostatectomy in patients with localized prostate cancer: Estimations of the number needed to treat according to tumor and patient characteristics. J Urol 188: 73-83, 2012.

6. Molitierno J, Evans A, Mohler JL, Wallen E, Moore D and Pruthi RS: Characterization of biochemical recurrence after radical prostatectomy. Urol Int 77: 130-134, 2006.

7. Shariat SF, Karakiewicz PI, Roehrborn CG and Kattan MW: An updated catalog of prostate cancer predictive tools. Cancer 113: 3075-3099, 2008.

8. Stephenson AJ, Scardino PT, Eastham JA, Bianco FJ Jr, Dotan ZA, DiBlasio CJ, Reuther A, Klein EA and Kattan MW: Postoperative nomogram predicting the 10 -year probability of prostate cancer recurrence after radical prostatectomy. J Clin Oncol 23: 7005-7012, 2005.

9. Lilja H, Ulmert D and Vickers AJ: Prostate-specific antigen and prostate cancer: Prediction, detection and monitoring. Nat Rev Cancer 8: 268-278, 2008.
10. Velonas VM, Woo HH, dos Remedios CG and Assinder SJ: Current status of biomarkers for prostate cancer. Int J Mol Sci 14: 11034-11060, 2013.

11. Liu L, McKeehan WL, Wang F and Xie R: MAP1S enhances autophagy to suppress tumorigenesis. Autophagy 8: 278-280, 2012.

12. Jiang X, Zhong W, Huang H, He H, Jiang F, Chen Y, Yue F, Zou J, Li X, He Y, et al: Autophagy defects suggested by low levels of autophagy activator MAP1S and high levels of autophagy inhibitor LRPPRC predict poor prognosis of prostate cancer patients. Mol Carcinog 54: 1194-1204, 2015.

13. Smith JS, Tachibana I, Pohl U, Lee HK, Thanarajasingam U, Portier BP, Ueki K, Ramaswamy S, Billings SJ, Mohrenweiser HW, et al: A transcript map of the chromosome 19q-arm glioma tumor suppressor region. Genomics 64: 44-50, 2000.

14. Yang P, Kollmeyer TM, Buckner K, Bamlet W, Ballman KV and Jenkins RB: Polymorphisms in GLTSCR1 and ERCC2 are associated with the development of oligodendrogliomas. Cancer 103: 2363-2372, 2005.

15. Neville PJ, Conti DV, Krumroy LM, Catalona WJ, Suarez BK, Witte JS and Casey G: Prostate cancer aggressiveness locus on chromosome segment 19q12-q13.1 identified by linkage and allelic imbalance studies. Genes Chromosomes Cancer 36: 332-339, 2003.

16. Slager SL, Schaid DJ, Cunningham JM, McDonnell SK, Marks AF, Peterson BJ, Hebbring SJ, Anderson S, French AJ and Thibodeau SN: Confirmation of linkage of prostate cancer aggressiveness with chromosome 19q. Am J Hum Genet 72: 759-762, 2003.

17. Epstein JI, Feng Z, Trock BJ and Pierorazio PM: Upgrading and downgrading of prostate cancer from biopsy to radical prostatectomy: Incidence and predictive factors using the modified Gleason grading system and factoring in tertiary grades. Eur Urol 61: 1019-1024, 2012.

18. Partin AW, Kattan MW, Subong EN, Walsh PC, Wojno KJ, Oesterling JE, Scardino PT and Pearson JD: Combination of prostate-specific antigen, clinical stage, and gleason score to predict pathological stage of localized prostate cancer. A multi-institutional update. JAMA 277: 1445-1451, 1997.

19. Xie ZC, Li TT, Gan BL, Gao X, Gao L, Chen G and Hu XH: Investigation of miR-136-5p key target genes and pathways in lung squamous cell cancer based on TCGA database and bioinformatics analysis. Pathol Res Pract 214: 644-654, 2018.

20. Guo Z, Chen X, Du T, Zhu D, Lai Y, Dong W, Wu W, Lin C, Liu L and Huang H: Elevated levels of epithelial cell transforming sequence 2 predicts poor prognosis for prostate cancer. Med Oncol 34: 13, 2017.

21. Zhang KX, Firus J, Prieur B, Jia W and Rennie PS: To die or to survive, a fatal question for the destiny of prostate cancer cells after androgen deprivation therapy. Cancers (Basel) 3: 1498-1512, 2011.

22. Rahman S, Sowa ME, Ottinger M, Smith JA, Shi Y, Harper JW and Howley PM: The Brd4 extraterminal domain confers transcription activation independent of pTEFb by recruiting multiple proteins, including NSD3. Mol Cell Biol 31: 2641-2652, 2011.

23. Adam K,Lambert M,Lestang E,Champenois G, Dusanter-Fourt I, Tamburini J, Bouscary D, Lacombe C, Zermati Y and Mayeux P: Control of Pim2 kinase stability and expression in transformed human haematopoietic cells. Biosci Rep 35: e00274, 2015.

24. Xu J, Zhang T, Wang T, You L and Zhao Y: PIM kinases: An overview in tumors and recent advances in pancreatic cancer. Future Oncol 10: 865-876, 2014.

25. Yu Z, Zhao X, Huang L, Zhang T, Yang F, Xie L, Song S, Miao P, Zhao L, Sun X, et al: Proviral insertion in murine lymphomas 2 (PIM2) oncogene phosphorylates pyruvate kinase M2 (PKM2) and promotes glycolysis in cancer cells. J Biol Chem 288: 35406-35416, 2013.

26. Ren K, Gou X, Xiao M, Wang M, Liu C, Tang Z and He W: The over-expression of Pim-2 promote the tumorigenesis of prostatic carcinoma through phosphorylating eIF4B. Prostate 73: 1462-1469, 2013.

27. Bohensky J, Shapiro IM, Leshinsky S, Watanabe $H$ and Srinivas V: PIM-2 is an independent regulator of chondrocyte survival and autophagy in the epiphyseal growth plate. J Cell Physiol 213: 246-251, 2007.

28. Amaravadi R, Kimmelman AC and White E: Recent insights into the function of autophagy in cancer. Genes Dev 30: 1913-1930, 2016. 